\title{
Voltage linear transformation circuit design
}

Lucas R. W. Sanchez, Moon-Seob Jin, R. Phillip Scott, Ryan J. Luder, Michael Hart

Lucas R. W. Sanchez, Moon-Seob Jin, R. Phillip Scott, Ryan J. Luder, Michael Hart, "Voltage linear transformation circuit design," Proc. SPIE 10401, Astronomical Optics: Design, Manufacture, and Test of Space and Ground Systems, 1040115 (5 September 2017); doi: 10.1117/12.2276166

SPIE Event: SPIE Optical Engineering + Applications, 2017, San Diego, California, United States 


\title{
Voltage Linear Transformation Circuit Design
}

\author{
Lucas R.W. Sanchez, Moon-Seob Jin, R. Phillip Scott, Ryan J. Luder, and Michael Hart \\ College of Optical Sciences, University of Arizona, 1630 E. University Blvd, Tucson, AZ 85721, \\ USA
}

\begin{abstract}
Many engineering projects require automated control of analog voltages over a specified range. We have developed a computer interface comprising custom hardware and MATLAB code to provide real-time control of a Thorlabs adaptive optics (AO) kit. The hardware interface includes an op amp cascade to linearly shift and scale a voltage range. With easy modifications, any linear transformation can be accommodated. In AO applications, the design is suitable to drive a range of different types of deformable and fast steering mirrors (FSM's). Our original motivation and application was to control an Optics in Motion (OIM) FSM which requires the customer to devise a unique interface to supply voltages to the mirror controller to set the mirror's angular deflection. The FSM is in an optical servo loop with a wave front sensor (WFS), which controls the dynamic behavior of the mirror's deflection. The code acquires wavefront data from the WFS and fits a plane, which is subsequently converted into its corresponding angular deflection. The FSM provides $\pm 3^{\circ}$ optical angular deflection for a $\pm 10 \mathrm{~V}$ voltage swing. Voltages are applied to the mirror via a National Instruments digital-to-analog converter (DAC) followed by an op amp cascade circuit. This system has been integrated into our Thorlabs AO testbed which currently runs at $11 \mathrm{~Hz}$, but with planned software upgrades, the system update rate is expected to improve to $500 \mathrm{~Hz}$. To show that the FSM subsystem is ready for this speed, we conducted two different PID tuning runs at different step commands. Once $500 \mathrm{~Hz}$ is achieved, we plan to make the code and method for our interface solution freely available to the community.
\end{abstract}

Keywords: Wavefront Sensor, Adaptive Optics, Laboratory Education, Circuit Design, Fast Steering Mirror

\section{INTRODUCTION}

Imaging through turbulence is an inescapable reality for any ground based telescope, and severely degrades image resolution. The larger the primary mirror, the worse is the impact on the resolution with respect to the diffraction limit. To correct aberrations caused by atmospheric turbulence, AO is necessary. AO systems typically contain three main components: a wave-front sensor (WFS), deformable mirror (DM), and fast steering mirror (FSM). These three components are interfaced through servo loops which usually operate at kilohertz rates, driven by a control computer. Continuous advances in AO technology over the past 40 years as well as reductions in cost are leading to an increasing number of applications. ${ }^{2}$ In the university environment, this manifests in a rising demand for educational tools. For these reasons, we believe it is important for our group to develop a functional AO system as a teaching device and a research instrument. Our group has acquired an AO kit from Thorlabs, which comprises a diode laser, 1:1 relays, a Shack-Hartmann WFS, a pellicle beam splitter, and a Boston Micromachines (BMC) 140actuator Multi-DM, as well as all the necessary mounts. We have modified our kit to include an Optics in Motion (OIM) FSM to handle larger wave front tilt errors than cannot be addressed by the relatively small stroke of the actuators on the DM alone, and to expand the range of AO technologies that may be demonstrated in the teaching laboratory.

Both the FSM and DM are controlled by a computer and some analog circuitry. The FSM provides $\pm 3^{\circ}$ optical angular deflection for a $\pm 10 \mathrm{~V}$ voltage swing. The FSM circuitry we designed for the kit was an op amp cascade which linearly shifts a $0-5 \mathrm{~V}$ voltage range output from a conventional digital-to-analog converter (DAC) card to $\pm 2.5 \mathrm{~V}$. Two independent channels of the circuit control negative and positive tip and tilt. Additionally, the circuit can be modified to control any voltage range, and can be linearly shifted to interface to different DACs and future adaptive components we expect to fabricate in our group. ${ }^{5}$

We have also written our own custom control software for the kit to maximize its flexibility and productivity as a pedagogical tool. ${ }^{3}$ The real-time components of the software are written in $\mathrm{C}++$ with an interface to a Matlab engine

Astronomical Optics: Design, Manufacture, and Test of Space and Ground Systems, edited by Tony B. Hull, Dae Wook Kim, Pascal Hallibert, Gary B. Hughes, Ronald G. Pirich, Proc. of SPIE Vol. 10401, $1040115 \cdot$ ? 2017 SPIE · CCC code: 0277-786X/17/\$18 - doi: 10.1117/12.2276166 
for data visualization, and graphical user interface (GUI). Components of Thorlab's AO software developers' kit (SDK) are used for data acquisition and hardware commands for the WFS, DM, and FSM.

In this paper, we describe the optical arrangement of the AO kit and provide the details of the circuit design used to drive the FSM. Additionally, we will present results of the system PID tuned to two different step responses. Lastly, we will discuss current and future work on developing the AO kit as a teaching and research tool.

\section{METHOD}

\subsection{AO System Design}

The optical layout of the modified AO kit, including the FSM, is shown in Figure 1. The AO kit accepts a $3 \mathrm{~mm}$ diameter beam whose aperture (located at the front of the diode laser) is at the front focal plane of the first lens in the system. The pupil, or the image of the aperture, is relayed using a Keplerian relay with $4 / 3$ magnification. The FSM, DM, and WFS all lie in conjugate pupil planes to properly read and correct phase aberration. As Figure 1 shows, the first adaptive component in the beam train is the FSM where the beam's tip/tilt is corrected. Next, the beam hits the DM, and higher-order aberrations are corrected. Finally, the pupil is relayed to the WFS and a CCD scoring camera. Figure 2 shows the behavior of the servo loop. First the local tip/tilt is calculated from the WFS spot field. The mean tip/tilt over the pupil is applied to the FSM and the higher order aberrations applied to the DM.

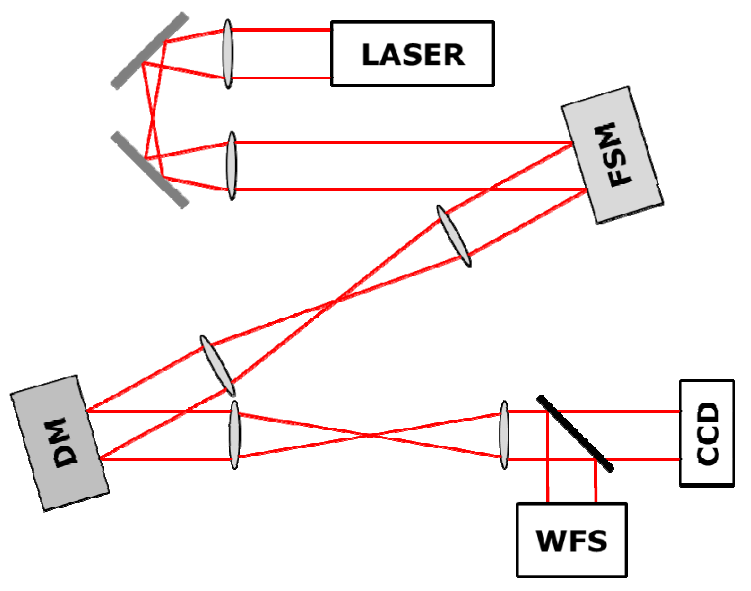

Figure 1. AO system scheme.

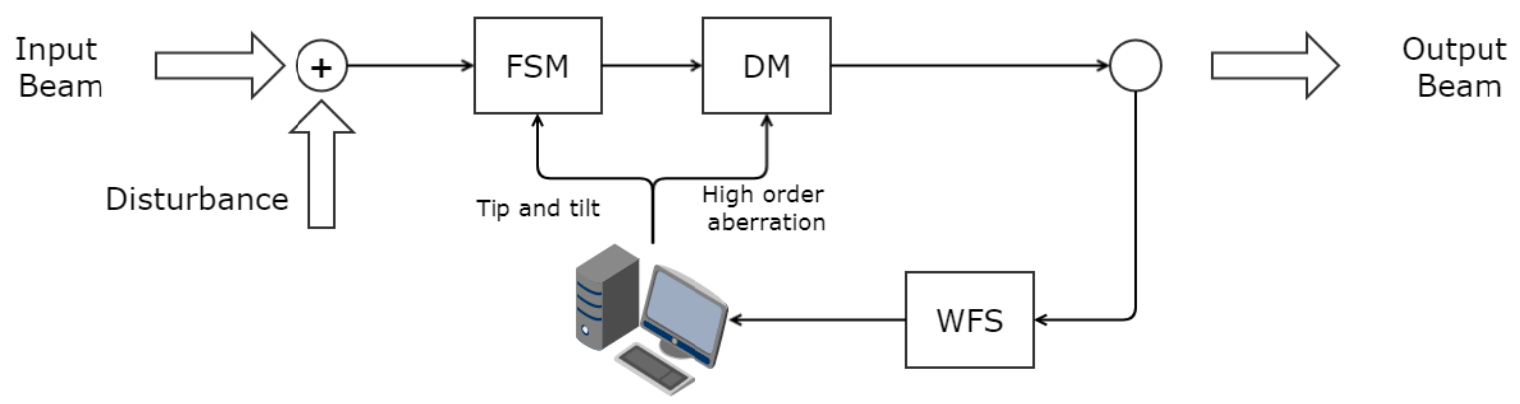

Figure 2. Beam control servo loop flowchart.

\subsection{FSM Interface}

\subsubsection{Circuit Theory and Design}

Our OIM FSM's $\mathrm{x} / \mathrm{y}$ angular deflection is controlled by inputting a voltage anywhere from $-10 \mathrm{~V}$ to $+10 \mathrm{~V}$ which corresponds to a $\pm 3^{\circ}$ optical deflection. Unfortunately, OIM only produces the FSM and not the FSM interface, 
which leaves the user to devise their own custom solution. The most common solution for interfacing with these FSMs are DACs. DACs that output negative voltages are more expensive than those that do not. A common output voltage range for inexpensive commercially available DAC cards is $0-5 \mathrm{~V}$. Thus, a custom hardware solution was necessary to linearly shift the voltage range to be centered on zero. The required optical motion of the FSM in the $\mathrm{AO}$ kit is less than $1^{\circ}$. Hence, unit amplification of the DAC signal to a range of $\pm 2.5 \mathrm{~V}$ was sufficient. Further amplification to the full range of the FSM would be straightforward, but would bring no optical advantage, and would reduce the precision of tilt control because of digitization noise. The circuit for a single channel of the amplifier is illustrated in Figure 3.

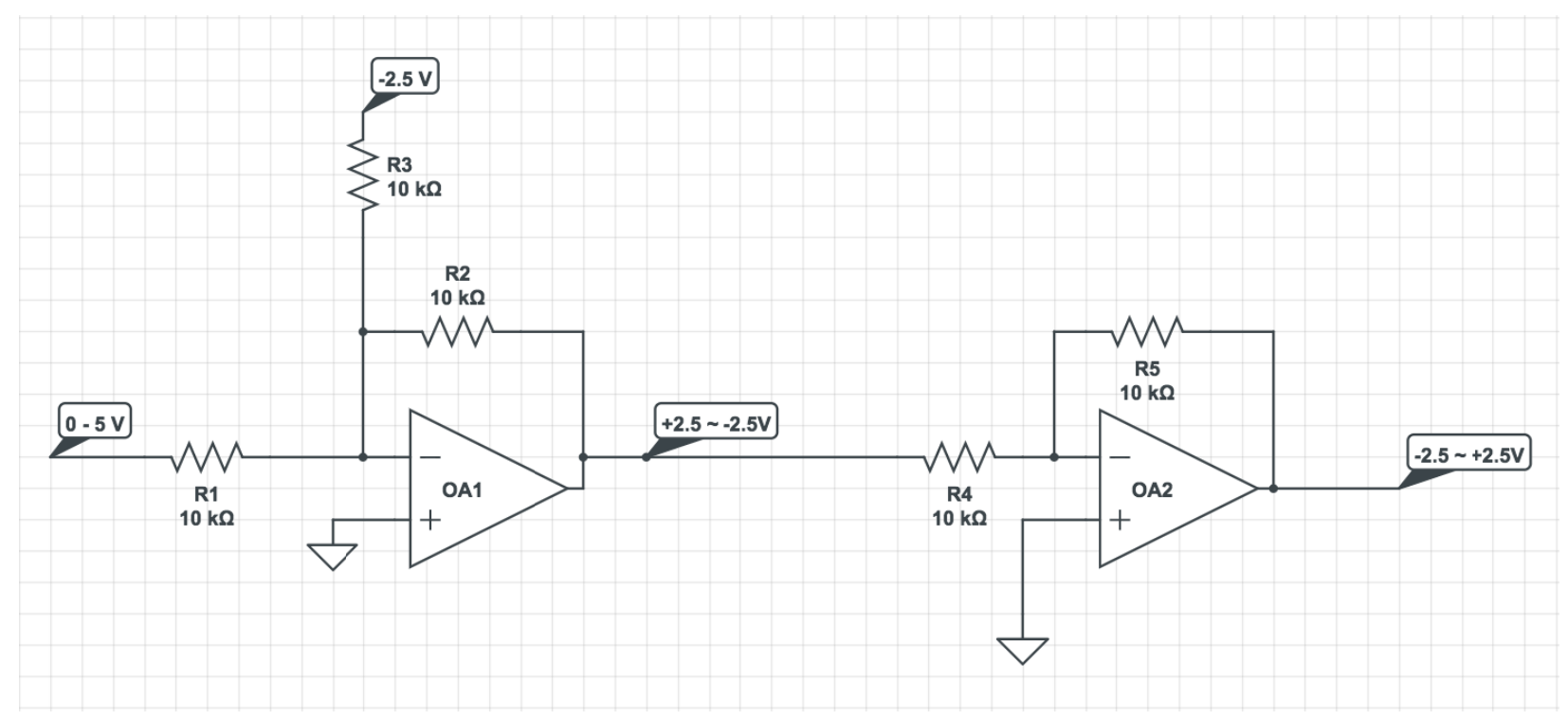

Figure 3. Voltage linear transformation circuit.

The first stage of the op amp cascade works as a summing amplifier whose circuit diagram and voltage equation is shown in Figure $4:^{4}$

\section{Summing Op-amp}
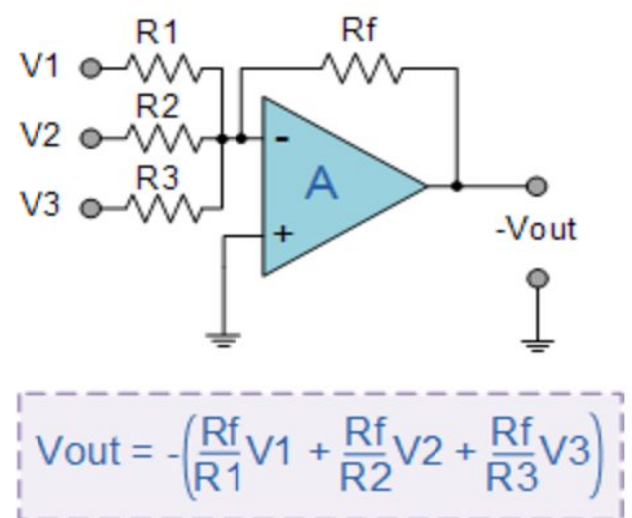

Figure 4. Summing amplifier.

For our case, all resistance ratios in Figure 4 are unity, and V3 does not exist, and the other voltage (aside from the input voltage) being summed is $-2.5 \mathrm{~V}$. Therefore, our equation for $\mathrm{V}_{\text {out }}$ is: 


$$
\mathrm{V}_{\text {out }}=2.5-\mathrm{V}_{\text {in }}
$$

The second stage of the amplifier is an inverting op-amp circuit which is shown in Figure 5 (from Ref 4). For our case, the ratio of the resistance values is unity, and therefore our equation for $\mathrm{V}_{\text {out }}$ is:

$$
\mathrm{V}_{\text {out }}=-\mathrm{V}_{\text {in }}
$$

By cascading these circuits, we obtain the following relation for the input/output voltages, which is also confirmed through experimentation:

$$
\mathrm{V}_{\text {out }}=\mathrm{V}_{\text {in }}+\mathrm{V}_{\text {ref }}
$$

\section{Inverting Op-amp}

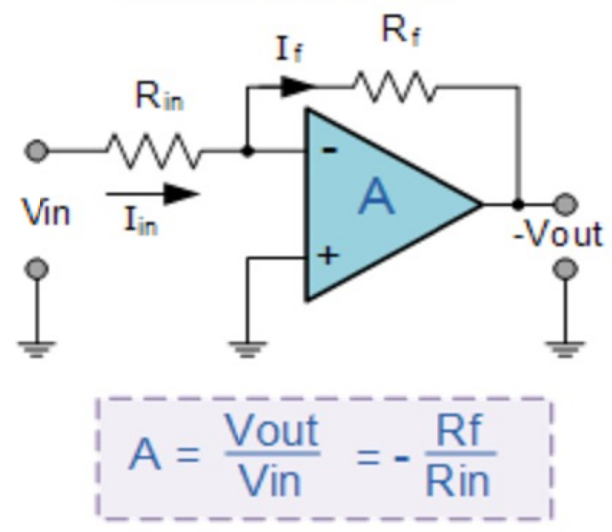

Figure 5. Inverting op-amp.

Theoretically, we can accommodate any voltage range, and will need to for future work in our lab. By adjusting the resistance values in the inverting op amp stage (Figure 5), we would be able to expand the output voltage range. For example, if we made the feedback resistor $\left(\mathrm{R}_{\mathrm{f}}\right) 20 \mathrm{k} \Omega$ the voltage range would instead be linearly shifted by the following relation where $\mathrm{V}_{\text {ref }}=-2.5 \mathrm{~V}$.

$$
\mathrm{V}_{\text {out }}=2\left(\mathrm{~V}_{\text {in }}+\mathrm{V}_{\text {ref }}\right)
$$

The symbolic relationship for these parameters is the following equation where the resistors subscripts are denoted in Figure 3:

$$
V_{\text {out }}=\left(\frac{R_{5}}{R_{4}}\right)\left(\frac{R_{2}}{R_{1}} V_{\text {in }}+\frac{R_{2}}{R_{3}} V_{\text {ref }}\right)
$$

\subsection{PID Tuning}

\section{EXPERIMENT}

The mirror's controller, shown in Figure 6, contains 2 sets of 3 potentiometers whose setting controls the respective PID gain for both the axes. The respective BNC connections for position and command for both axes are shown. 


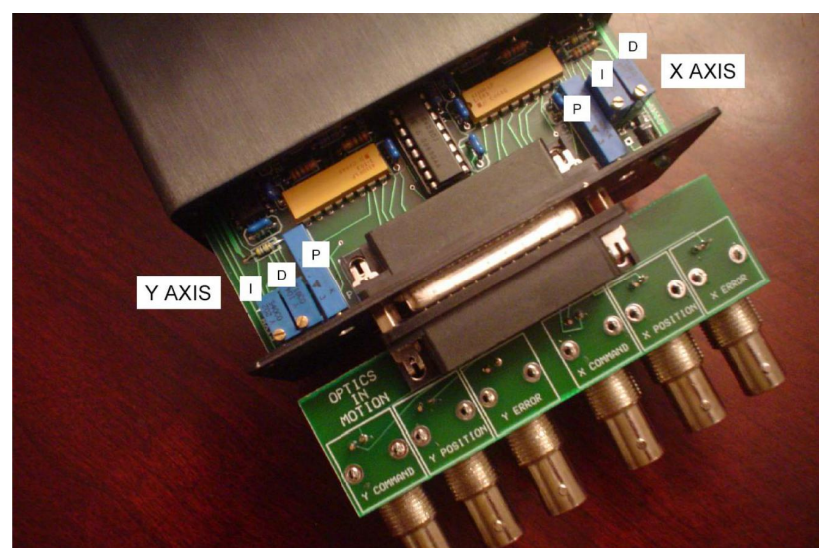

Figure 6. Mirror controller box

To show that the FSM subsystem is capable of use at $500 \mathrm{~Hz}$, we sought to tune the PID control for typical step commands and analyze the mirrors step response. The goal of PID tuning is to minimize the rise time, steady-state error, settling time, and fractional overshoot between the signal and the position as illustrated in Figure 7.

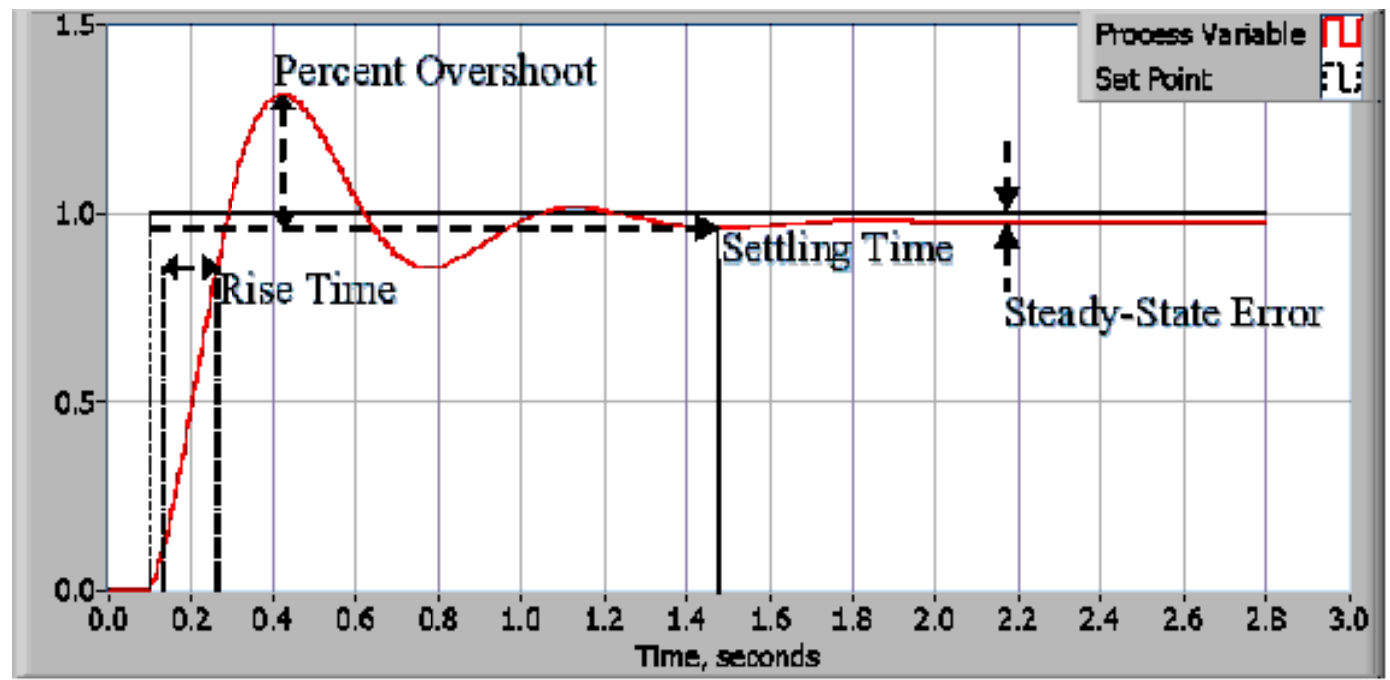

Figure 7. PID tuning vocabulary. ${ }^{6}$

The system required to tune the FSM response contains the following components: FSM, function generator, oscilloscope, and necessary connections. A picture of the experiment setup lies below: 


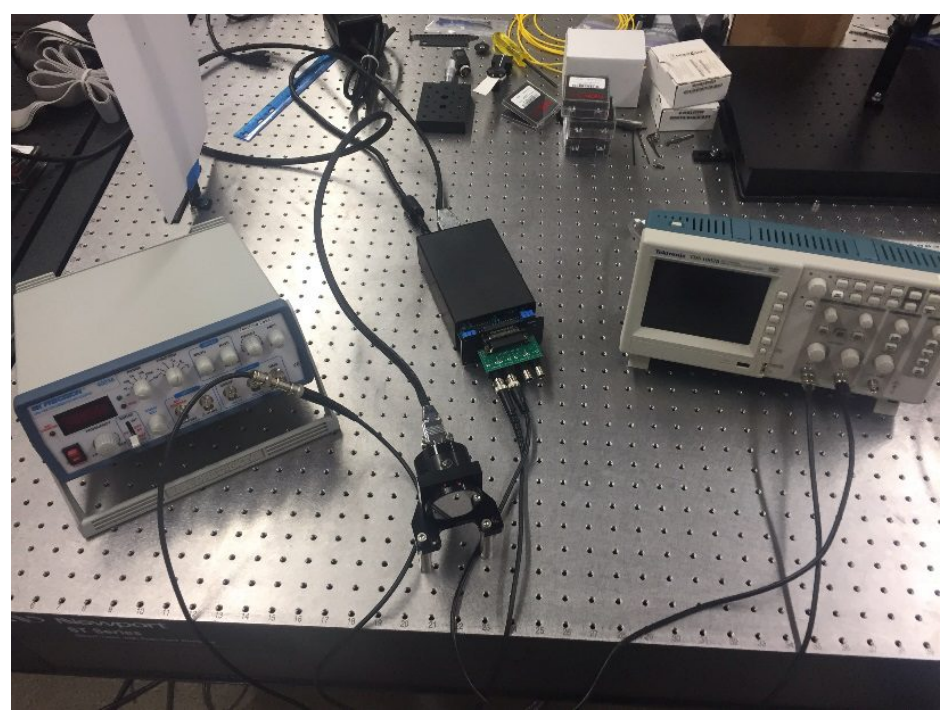

Figure 8. PID tuning set-up

We began by commanding the mirror with a saw tooth wave, and analyzed the mirror's step response. We were specifically concerned with having a short rise time with negligible overshoot. Steady-state error proved to be negligible at this point in our investigation. Since our prime motivation for this experiment was to analyze the mirror's behavior at the typical step responses it would experience in operation, we first had to derive the expected atmospheric tilt error the mirror would have to compensate. We intend to deploy the system at the Kuiper $1.5 \mathrm{~m}$ telescope on Mt. Bigelow, AZ. The typical variance of single-axis wave front tilt in radians is given by the following formula: ${ }^{1}$

$$
\sigma_{\text {Tilt }}^{2}=0.184\left(\frac{D}{r_{0}}\right)^{5 / 3}\left(\frac{\lambda}{D}\right)^{2}
$$

where $D$ is the telescope diameter, $\lambda$ is the wavelength of observation, and $r_{0}$ is the atmospheric coherence length. The typical night-time value of $r_{0}$ atop Mt. Bigelow is about $10 \mathrm{~cm}$ at $500 \mathrm{~nm}$. Atmospheric tilt that the primary mirror sees is magnified by reimaging optics, so to find the required stroke $S_{T i l t}$ of the FSM we use the following equation: ${ }^{1}$

$$
S_{\text {Tilt }}=\frac{1}{2}\left(2.5 \sigma_{\text {Tilt }}\right) M
$$

where $M$ is the angular magnification between the primary mirror and the FSM, and we assume a maximum stroke of $2.5 \times$ the standard deviation. From this equation, we find that the required stroke for the FSM is approximately $.003^{\circ}$ which corresponds to a $20 \mathrm{mV}$ input. This is a relatively small range compared to the mirror's full potential range. This is a rather small voltage by which to assess a step amplitude, so we elected to command the mirror to go an order of magnitude above this maximum expected stroke, and oscillate between approximately $\pm 225 \mathrm{mV}$. The final step response after PID tuning is shown in Figure 9. 


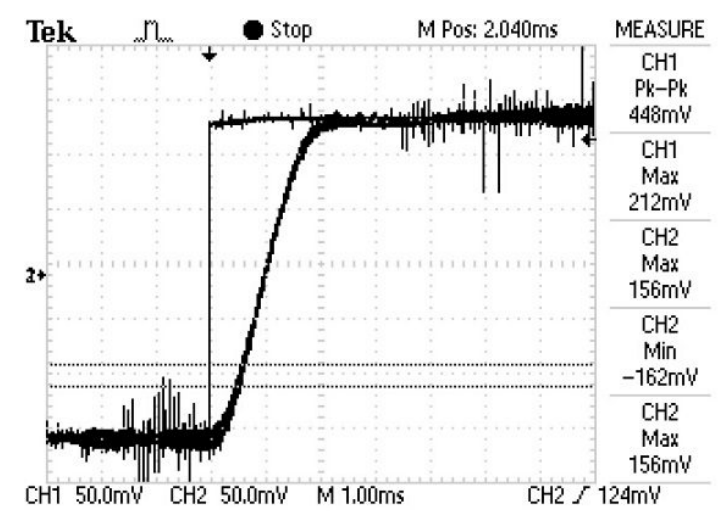

Push an option button to change its measurement

Figure 9. PID tuned response of the FSM to a step-function command showing a rise time of approximately $2 \mathrm{~ms}$.

The FSM responds with a rise time of $2 \mathrm{~ms}$ with no overshoot and rapid settling time. This leads us to be confident that the mirror is capable of operation at $500 \mathrm{~Hz}$. We have also tested the mirror at roughly $\pm 3.5 \mathrm{~V}$ which corresponds to approximately $0.525^{\circ}$. This was done to test the mirror's correction speed at larger stroke. We began by setting all three gains to 0 , with the result as shown in Figure 10.

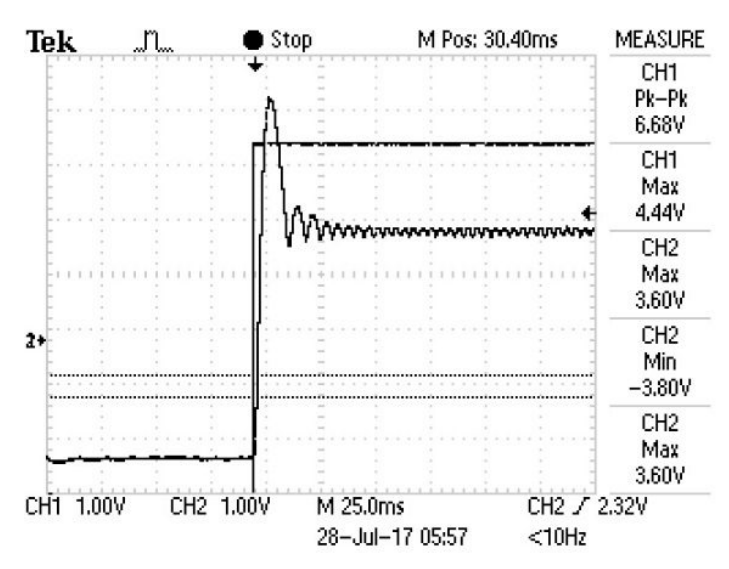

Figure 10. Mirror's step response to a large voltage swing with all PID gains set to zero.

Optimization proceeded with sequential adjustment of the proportional, derivative, and integral gain terms. Final joint optimization of all three led to reduced integral gain and increased proportional gain. The results at each step are shown in Figure 11. Even at this large amplitude, the rise time is about $5 \mathrm{~ms}$, implying that the mirror can be operated at $200 \mathrm{~Hz}$. 

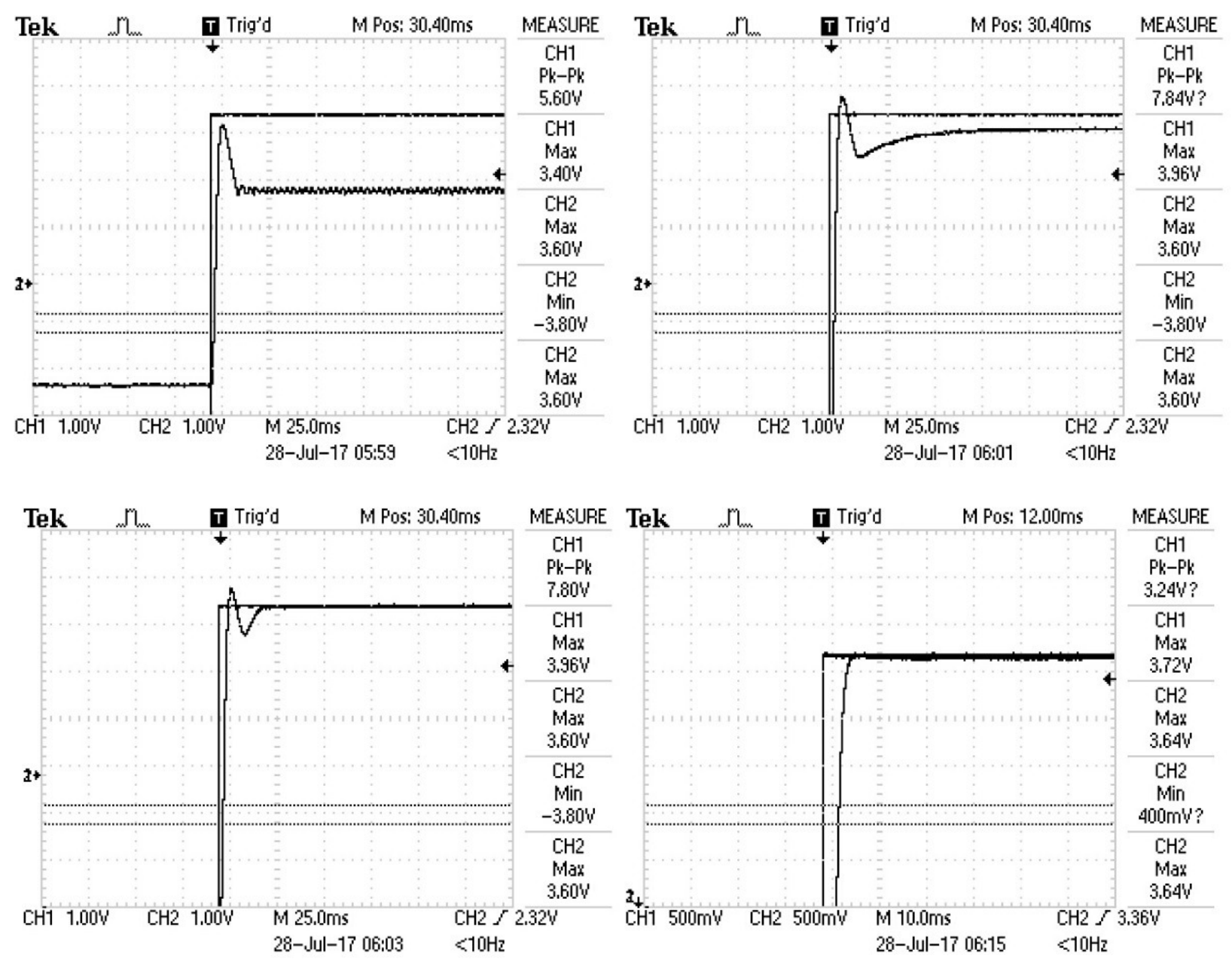

Figure 11. FSM step response after successive adjustment of proportional (top left), derivative (top right), and integral (bottom left) gains, followed by joint optimization (bottom right).

\subsection{Parallel AO Kit Investigations}

The AO kit contains a $635 \mathrm{~nm}$ collimated laser diode, a WFS pre-built by Thorlabs, BMC Multi-DM, a pellicle beam splitter, OIM FSM, a phase plate to simulate turbulence, as well as all the necessary relay optics and mounts. The arrangement is shown in Figure 12. The software to run the system is a mix of C++ drivers and MATLAB to implement the control loop. While computationally inefficient, the architecture is ideal for teaching purposes since it exposes the functional components of the loop control in a format that is amenable to ready experimentation.

We have shown that the FSM can handle a typical step response at $500 \mathrm{~Hz}$. The full AO kit's present capability is described in Jin et al. ${ }^{3}$ The high-order correction loop is currently limited to $11 \mathrm{~Hz}$ by the software, two orders of magnitude slower than the speeds necessary for astronomical applications. This is principally because of excessive time devoted to data transmission between the MATLAB and $\mathrm{C}++$ components of the code. A second version of the code, entirely in $\mathrm{C}++$, is under development to support operation on sky. 


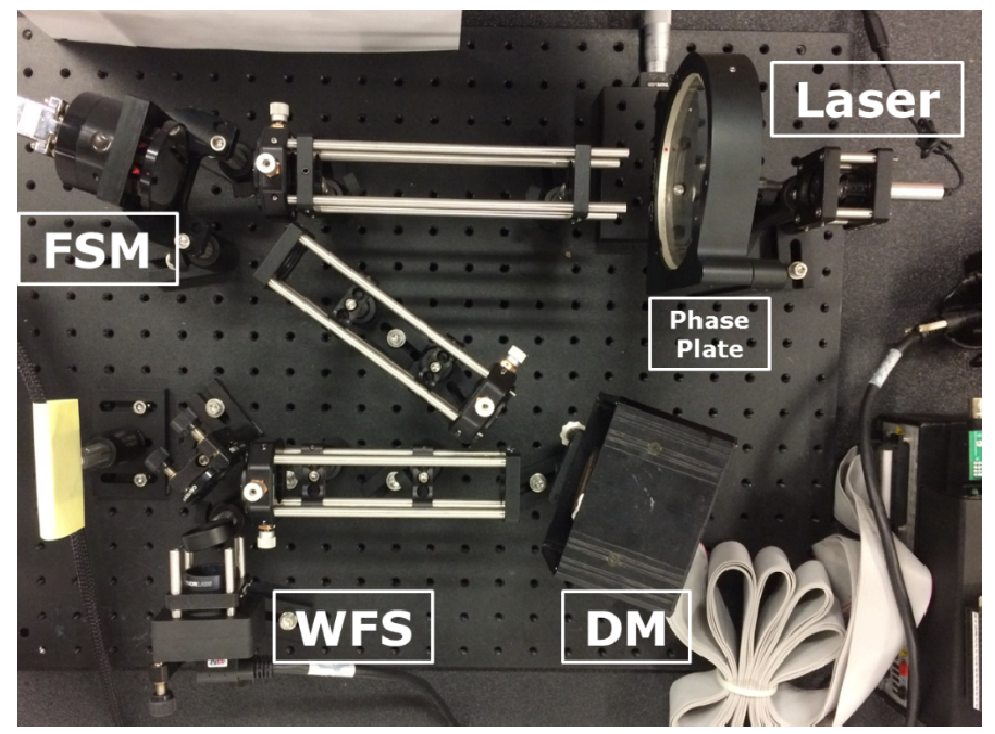

Figure 12. AO system setup on the optical bench.

\section{FUTURE WORK}

Eventually this system will be used in a teaching lab and with preliminary success at $11 \mathrm{~Hz}$ speeds, its trial run in that capacity will be in the fall 2017 semester at the University of Arizona. The system supports the exploration of AO correction in many ways: with and without the use of a FSM, wavefront control through zonal or modal reconstruction, the effects of noise, variations in the spatial and temporal coherence of the aberration, and the servo controller itself. ${ }^{3}$ Aside from developing the system as a teaching device, we also plan to use the system as an AO test bench at the Kuiper $1.5 \mathrm{~m}$ telescope on Mt. Bigelow near Tucson, AZ. Lastly, we plan to use a voltage shifting circuit similar to the one described here to control a new prototype DM with a ceramic facesheet that we are fabricating in our laboratory. ${ }^{5}$

\section{REFERENCES}

1. Tyson, R. K., Frazier, B. W., Field Guide to Adaptive Optics, SPIE press, Bellingham, Washington, 1-84 (2012)

2. Boston Micromachines Corporation, Adaptive Optics 101: Overview, Tech Review \& Applications Introduction and Motivation, Boston Micromachines Corporation, Cambridge, Massachusetts, 1-11, (2014)

3. M. Jin, R. Luder, L. Sanchez, and M. Hart, "Control code for laboratory adaptive optics teaching system," Proc. SPIE 10401-52 (2017)

4. Electronics Tutorials, http://www.electronics-tutorials.ws/opamp/opamp 8.html

5. J. A. Rice, M. Hart E. Corral, X. Gou, D. W. Kim, "Fabrication and Implementation of a New Ceramic Material in an Adaptive Optics System," J. Opt. Fab. Test. 2017, 1-2 (2017)

6. National Instruments, http://www.ni.com/white-paper/3782/en/ 